\title{
New vaccine strategies against enterotoxigenic Escherichia coli. II: Enhanced systemic and secreted antibody responses against the CFA/I fimbriae by priming with DNA and boosting with a live recombinant Salmonella vaccine
}

M.O. Lásaro ${ }^{1}$ A.M.B. Alves ${ }^{1,2}$,

H.C.R. Guillobel ${ }^{3}$,

D.F. Almeida ${ }^{1}$ and

L.C.S. Ferreira ${ }^{1}$
${ }^{1}$ Laboratório de Fisiologia Celular, Instituto de Biofísica Carlos Chagas Filho, Universidade Federal do Rio de Janeiro, Rio de Janeiro, RJ, Brasil ${ }^{2}$ Laboratório de Tecnologia Bacteriana, Bio-Manguinhos, Fundação O swaldo Cruz, Rio de Janeiro, RJ, Brasil

${ }^{3}$ Departamento de Biofísica e Biometria, Instituto de Biologia, Universidade Estadual do Rio de Janeiro, Rio de Janeiro, RJ, Brasil

\section{Correspondence}

L.C.S. Ferreira

Laboratório de Fisiologia Celular Instituto de Biofísica, UFRJ 21941-590 Rio de Janeiro, RJ Brasil

Fax: +55-21-280-8193

E-mail: Icsf@ibccf.biof.ufrj.br

Presented at the International Symposium "The Third Revolution on Vaccines: DNA Vaccines", Belo Horizonte, MG, Brasil,

November 3-7, 1997.

Research supported by CAPES, CNPq, FAPERJ, PADCT and PRONEX.

Received November 24, 1998 Accepted December 9, 1998

\section{Abstract}

The induction of systemic (IgG) and mucosal (IgA) antibody responses against the colonization factor I antigen (CFA/I) of enterotoxigenic Escherichia coli (ETEC) was evaluated in mice primed with an intramuscularly delivered CFA/I-encoding DNA vaccine followed by two oral immunizations with a live recombinant Salmonella typhimurium vaccine strain expressing the ETEC antigen. The booster effect induced by the oral immunization was detected two weeks and one year after the administration of the DNA vaccine. The DNAprimed/Salmonella-boosted vaccination regime showed a synergistic effect on the induced CFA/I-specific systemic and secreted antibody levels which could not be attained by either immunization strategy alone. These results suggest that the combined use of DNA vaccines and recombinant Salmonella vaccine strains can be a useful immunization strategy against enteric pathogens.

\section{Introduction}

Stimulation of the mammalian immune system by intramuscular $(\mathrm{im})$ administration of plasmid DNA encoding heterologous antigens represents an exciting new immunization procedure which has been repeatedly shown to induce humoral and cellular im-
Key words

- DNA vaccine

- ETEC

- CFA/l fimbriae

- Salmonella bivalent vaccine mune responses, some of them protective upon challenge, against several pathogenic viruses, bacteria and parasites (1-3). Nonetheless, one of the main limitations of DNA vaccines has been the lack of an induced mucosal response, i.e., IgA isotype immunoglobulins produced on mucosal surfaces and in exocrine gland secretions. Secreted IgA 
represents the most efficient and effective immune barrier against a number of pathogens, such as enteric bacterial pathogens which use the intestinal mucosal surface to gain access to the mammalian host (4). This has led to the search for alternative experimental approaches to target DNA vaccines to mucosal immune system inductive sites such as direct intravaginal delivery (5), oral delivery of encapsulated DNA in biodegradable microspheres (6), particle bombardment of mucosal surfaces with gene gun-delivered DNA-coated gold particles $(7,8)$ or nasal administration of DNA-liposome complexes (9).

The combined use of parenteral (whole cell antigens) and oral (live bacterial cells) immunizations has been reported to enhance systemic and mucosal antibody responses in mice vaccinated against Shigella (10). Using a similar immunization strategy we evaluated the priming of the mucosal immune system by a parenteral DNA vaccine encoding the colonization factor I antigen (CFA/I) fimbrial adhesin subunit of enterotoxigenic Escherichia coli $(11,12)$ followed by oral immunization with a live recombinant Salmonella vaccine strain expressing the same enterotoxigenic Escherichia coli (ETEC) antigen (13). We show that the combined immunization regime results in enhanced production of the CFA/I-specific systemic and secreted antibody response.

\section{Material and Methods}

\section{DNA vaccine}

pRECFA, which encodes the ETEC CFA/ I subunit fused with herpes simplex virus glycoprotein D1 (HSV gD1) under the control of Rous sarcoma virus (RSV) promoter, was used for the DNA immunizations. The construction of pRECFA and its induced immune response following administration in mice have been reported $(11,12)$.

\section{Attenuated Salmonella vaccine}

The S. typhimurium HG3 strain is a nonreverting aromatic-dependent (aroA) histidine-requiring mutant transformed with pCFA-1, which encodes the ETEC $c f a B$ gene, as previously described (13). Before administration to mice, the Salmonella vaccine strain was cultivated at $37^{\circ} \mathrm{C}$ in Luria broth in the presence of $0.5 \mathrm{mM}$ IPTG for $4 \mathrm{~h}$ to induce expression of CFA/I. Cells were centrifuged, washed once and resuspended in phosphate buffered saline (PBS) to a final concentration of approximately $10^{10} \mathrm{cfu} / \mathrm{ml}$ and used the same day. Expression of CFA/ I by HG3 was confirmed by SDS-PAGE and Western blot.

\section{Immunization protocol and sample collection}

Four- to six-week-old male BALB/c mice purchased from Oswaldo Cruz Foundation (Fiocruz, Rio de Janeiro) were used in all experiments. For DNA vaccination mice were inoculated im with two 100- $\mu \mathrm{g}$ doses of pRECFA given two weeks apart as described (11). For immunization with Salmonella, mice were intragastrically inoculated using a blunt-tipped feeding needle with two doses of $10^{10} \mathrm{cfu}$ in $0.5 \mathrm{ml}$ of PBS given one week apart. Two groups of five mice were submitted to the combined immunization regime: the first (denoted young mice) received the first Salmonella vaccine dose two weeks following the last DNA immunization, while the second group (denoted old mice) was vaccinated with the recombinant Salmonella strain one year following the second DNA dose. Control groups were immunized only with two doses of HG3 or pRECFA or with two doses of pRE4, which did not contain the $c f a B$ gene insert, followed by two HG3 doses. Mice were bled by retro-orbital puncture before inoculation (preimmune sera) and two weeks after the last DNA immunization. One week after the last administration 
of HG3, animals were sacrificed and bled by cardiac puncture. Initially, serum samples were individually tested for reactivity against CFA/I subunits in an enzyme-linked immunosorbent assay (ELISA), then pooled and stored at $-20^{\circ} \mathrm{C}$ for subsequent analysis. For analysis of the mucosal immune response, feces were collected overnight and freeze dried. Fifteen pellets were homogenized in PBS (0.5 ml) and centrifuged at 10,000 rpm for $10 \mathrm{~min}$ at $4^{\circ} \mathrm{C}$, and the supernatant was collected for CFA/I-specific IgG IgA ELISA.

\section{Detection of antibodies by ELISA}

ELISA was carried out with purified heatdenatured CFA/I subunits as a solid-phase bound antigen prepared as described (14). CFA/I-specific IgG and IgA ELISA were carried out by standard procedures using sera and fecal extracts from immunized mice, respectively $(11,13)$. CFA/I-specific IgG titers were determined by end-point dilution in ELISA.

\section{Results}

Two groups of mice immunized $i m$ with two $100-\mu \mathrm{g}$ doses of pRECFA given two weeks apart presented systemic CFA/I-specific IgG titers which reached maximum levels two weeks after the last dose (average of $10^{4}$ ), followed by a declining phase of 20 weeks and later by the stabilization of anti- body levels with titers ranging from 1 to 2.5 $x 10^{3}$. In pRECFA-inoculated mice orally boosted with two doses of CFA/I-expressing live recombinant Salmonella HG3 cells 2 or 52 weeks after the last DNA dose, the antiCFA/I serum IgG levels rose to $2 \times 10^{5}$ and 6 $\mathrm{x} 10^{4}$, respectively (Figure 1). Animals immunized with only two doses of HG3 or with pRECFA alone presented reduced CFA/Ispecific systemic IgG levels in relation to those immunized with the combined DNAprimed/Salmonella-boosted protocol (Figure $1)$.

Mucosal anti-CFA/I IgA responses were monitored in both groups of vaccinated mice (young and old mice). As was the case for the systemic antibody responses, the combined use of DNA and recombinant Salmonella vaccines resulted in an enhanced secreted IgA response in fecal extracts (Figure 2 ). However, the booster effect of orally delivered CFA/I-expressing Salmonella was demonstrable only in young mice, i.e., those which were immunized two weeks after the last DNA dose. CFA/I-specific IgA levels in mice orally boosted with live Salmonella cells two weeks after the last DNA dose were at least five times higher than those observed in mice immunized with two doses of HG3 or pRECFA only. Mice immunized with two doses of HG3 had significantly higher CFA/I-specific IgA levels which did not differ from the values found in those immunized with the combined DNA-primed/

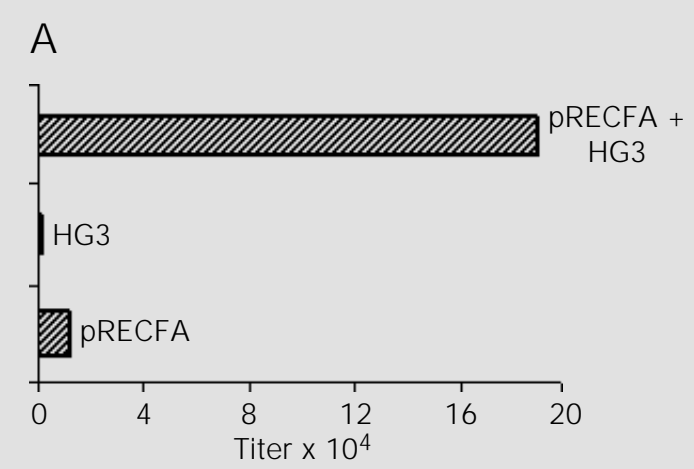

B

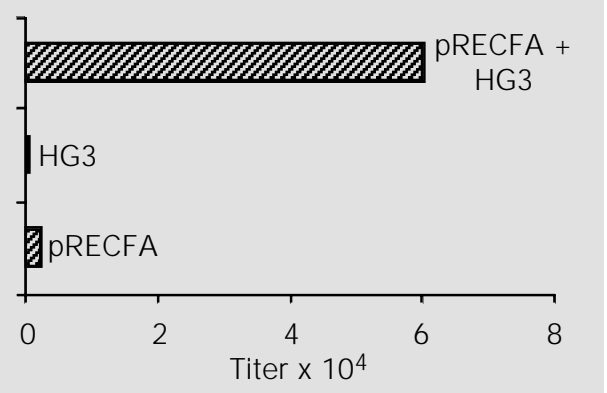

Figure 1 - Systemic IgG CFA/Ispecific antibody response in mice immunized im with PRECFA as primer and orally boosted with two doses of Salmonella vaccine strain (HG3). The doses of Salmonella vaccine were given two weeks (A) or 54 weeks (B) after the last DNA dose. Mice were immunized with the complete protocol (pRECFA + HG3), two pRECFA doses (pRECFA) or two HG3 doses. Column length indicates the mean titers of pooled senum samples $(\mathrm{N}=5)$. Standard deviations were less than $5 \%$ of the calculated mean values. 
Figure 2 - CFA/l-specific secreted IgA (slgA) antibody response in fecal extracts from mice immunized im with pRECFA as primer and orally boosted with two doses of Salmonella vaccine strain (HG3) detected by IgA-ELISA. The doses of Salmonella vaccine were given two weeks (A) or 54 weeks (B) after the last DNA dose. Mice were immunized with the complete protocol (pRECFA + HG3), two pRECFA doses (pRECFA) or two HG3 doses. Column length indicates the mean of duplicate absorbance values of pooled fecal extract samples $(\mathrm{N}=5)$ at $1: 4$ dilution. Standard deviations were less than $5 \%$ of the calculated mean values.

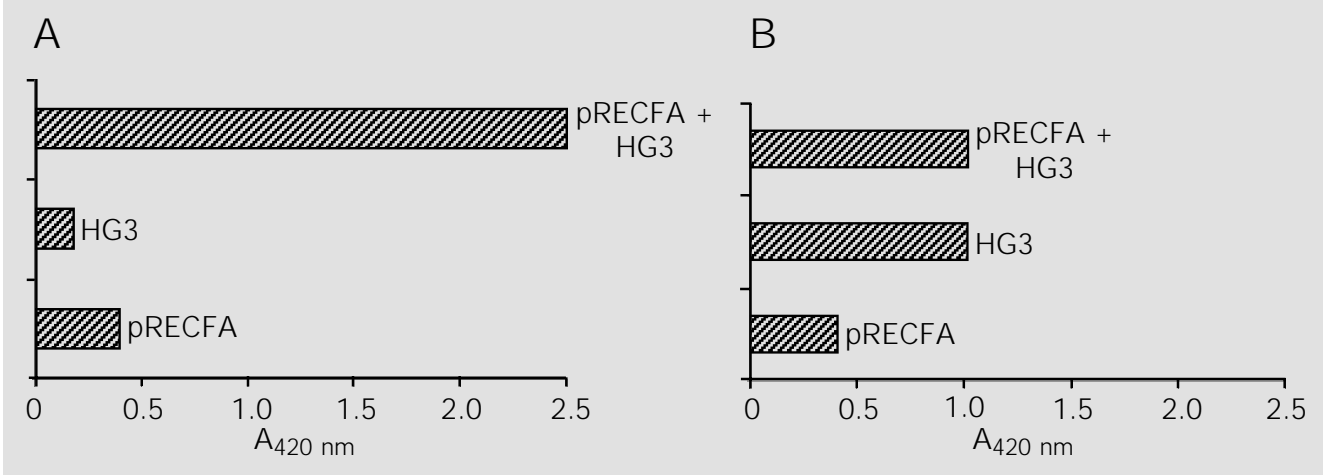

Salmonella-boosted protocol in fecal extracts one year after the last dose (Figure 2).

In contrast to serum raised against CFA/ I encoded by pRECFA (11), serum antibodies generated by the combined DNA-primed/ Salmonella-boosted regime inhibited the adhesive properties of CFA/I fimbriae expressed by ETEC cells, as evaluated in a hemagglutination inhibition assay (data not shown), in contrast to serum CFA/I-specific antibodies induced by pRECFA which were not inhibitory under similar conditions (11).

\section{Discussion}

In this study we analyzed the systemic and mucosal immune responses induced in mice immunized with a combined parenteral DNA-primed/oral Salmonella-boosted vaccination strategy. We observed, for the first time, that association of an im delivered DNA vaccine (primer) with a live recombinant attenuated Salmonella strain (booster) carrying a heterologous antigen (CFA/I) resulted in enhanced antigen-specific antibody production in both systemic (IgG) and mucosal (IgA) compartments. Moreover, our results indicated that, at least for systemic immune responses, the priming of the immune system by the DNA vaccine is a longlasting event since inoculated animals were able to respond to the oral administration of Salmonella cells up to 52 weeks after DNA immunization.
It has been shown that parenteral immunization with heat-killed Shigella cells results in enhanced systemic and mucosal immune responses following oral boostering with live cells (10). In the present study we showed that the same rationale could be applied to DNA vaccines. Using a DNA vaccine encoding the CFA/I ETEC fimbrial adhesin to prime the animals and a CFA/I expressing Salmonella vaccine strain as the oral boostering formulation we detected antibody levels in both serum and fecal extracts, which could not be attained by only one immunization strategy. Thus, such approach overcomes in part the deficient activation of mucosal immune responses by $i m$ delivered DNA vaccines since activation of IgA production could be efficiently triggered in DNA vaccinated mice following local stimulation with recombinant live Salmonella cells. Although several recent reports have indicated that direct stimulation of the mucosal immune system is feasible (5-9), the combined vaccination strategy described here does not require any specialized delivery method and takes advantage of both immunization protocols, i.e., the strong and long-term stimulation of antibody responses induced by DNA vaccines and the efficient targeting and stimulation of the mucosal immune system by oral immunization with live Salmonella cells.

Expression of secreted bacterial proteins such as toxins and adhesins by host cells 
transfected with DNA vaccines may lead to the production of altered proteins with regard to their native conformations. Such in vivo formed antigens may lead to the production of reduced antibody levels or inappropriate epitope specificity $(11,15)$. In both cases the protective efficacy of the vaccine would be seriously affected. Mice immunized with pRECFA have been shown to induce anti-CFA/I serum antibodies unable to inhibit the adhesive properties of intact CFA/I fimbriae due to altered epitope specificity (11). On the other hand, mice immunized with the combined DNA-primed/Salmonella-boosted protocol developed a systemic antibody response able to block the adhesive properties of CFA/I $\mathrm{I}^{+}$ETEC cells. These results suggest that the combination of DNA priming with oral Salmonella boosting could not only activate a quantitatively higher antibody response but also a qualitatively different response based on the epitope specificity of the induced antibodies.

Recently, ETEC was included by the
WHO among the top five enteric pathogens to be controlled by vaccination (16). At present only one ETEC vaccine, based on the oral delivery of an inactivated pool of ETEC strains expressing the most prevalent CFAs and purified heat-labile toxin $\beta$-subunit, is under testing for human use (17). The proposed combination of two different immunization strategies which could enhance both systemic and mucosal antibody responses against an important ETEC adhesin may represent a significant step towards the development of a vaccine against enteric bacterial pathogens.

\section{Acknowledgments}

We thank Eduardo Camacho and Celso Pereira for excellent technical assistance. We are also indebted to Dr. G. Cohen for the pRE4 vector and to Dr. A.M. Svennerholm for the ETEC strains and purified CFA/I fimbriae.

\section{References}

1. Ulmer J B, Donnelly J J, Parker SE, Rhodes GH, Felgner PL, Dwarki VJ , Gromkowski $\mathrm{SH}$, Ramdall Deck R, Dewitt CM, Friedman A, Hawe LA, Leander KR, Martinez D, Perry HC, Shilver J W, Montgomery DL \& Liu MA (1993). Heterologous protection against influenza by injection of DNA encoding viral protection. Science, 259: 1745-1749.

2. Robinson HL (1997). Nucleic acid vaccines: an overview. Vaccine, 15: 785-787.

3. Donnelly J J , Ulmer J B, Shiver J W \& Lui MA (1998). DNA vaccines. Annual Review of Immunology, 15: 617-648.

4. Levine MM (1987). Escherichia coli that cause diarrhea: enterotoxigenic, enteropathogenic, enteroinvasive, enterohemormagic and enteroadherent. J oumal of Infectious Diseases, 155: 377-389.

5. Wang B, Dang K, Agadjanyan MG, Srikantan V, Li F, Ugen KE, Boyer J , Merva M, Williams WV \& Weiner DB (1997). Mucosal immunization with a DNA vaccine induces immune responses against
HIV-1 at a mucosal site. Vaccine, 15: 821825.

6. J ones DH, Corris S, McDonald S, Clegg J CS \& Farrar GH (1997). Poly (DL-lactideco-glycolide)-encapsulated plasmid DNA elicits systemic and mucosal antibody responses to encoded protein after oral administration. Vaccine, 15: 814-817.

7. Fynan EF, Webster RG, Fuller DH, Haynes J R, Santoro JC \& Robinson HL (1993). DNA vaccines: protective immunization by parenteral, mucosal and gene-gun inoculations. Proceedings of the National Academy of Sciences, USA, 90: 1147811482.

8. Ban EM, van Gingkel FW, Simecka JW, Kiyono $\mathrm{H}$, Robinson $\mathrm{HL} \& \mathrm{McG}$ ee J R (1997). Mucosal immunization with DNA encoding influenza hemagglutinin. Vaccine, 15: 811-813.

9. Klavinskis LS, Gao L, Bamfield C, Lehner T \& Parker S (1997). Mucosal immunization with DNA-liposome complexes. Vaccine, 15: 818-820.
10. Karen FF, MacDonald RA \& Carey J L (1988). Combined parenteral and oral immunization results in an enhanced mucosal immunoglobulin A response to Shigella flexneri. Infection and Immunity, 56: 910-915.

11. Alves AMB, Lásaro MO, Almeida DF \& Ferreira LCS (1998). Epitope specificity of antibodies raised against enterotoxigenic Escherichia coli CFA/l fimbriae in mice immunized with naked DNA. Vaccine, 16: 9-15.

12. Alves $A M B$, Lásaro MO, Almeida DF \& Ferreira LCS (1999). New vaccine strategies against enterotoxigenic Escherichia coli. I: DNA vaccines against the CFA/I fimbrial adhesin. Brazilian J ournal of Medical and Biological Research, 32: 223-229.

13. Guillobel HCR, Luna MG, Camacho EF, Almeida DF \& Ferreira LCS (1998). Immunization against the colonization factor antigen I of enterotoxigenic Escherichia coli by administration of a bivalent Salmonella typhimurium aroA strain. Brazilian J oumal 
of Medical and Biological Research, 31: 545-554.

14. Luna MG, Ferreira LCS, Almeida DF \& Rudin A (1997). Peptides ${ }^{14} \mathrm{VIDLL}^{18}$ and ${ }^{96}$ FEAAAL ${ }^{101}$ defined as epitopes of antibodies raised against amino acid sequences of enterotoxigenic Escherichia coli colonization factor antigen I fused to Salmonella flagellin. Microbiology, 143:
3201-3207.

15. Saikh KU, Sesno J, Brandler P \& Ulrich RG (1998). Are DNA-based vaccines useful for protection against secreted bacterial toxins? Tetanus toxin test case. Vaccine, 16: 1029-1038.

16. Sansonetti PJ (1998). Slaying the Hydra all at once or head by head? New candidate vaccines show promise in protecting against the diverse pathogens that cause diarrheal diseases. Nature Medicine, 4: 499-500.

17. J ertborn $M$, Ahrén $C$, Holmgren J \& Svennerholm A-M (1998). Safety and immunogenicity of an oral inactivated enterotoxigenic Escherichia coli vaccine. vaccine, 16: 255-260. 